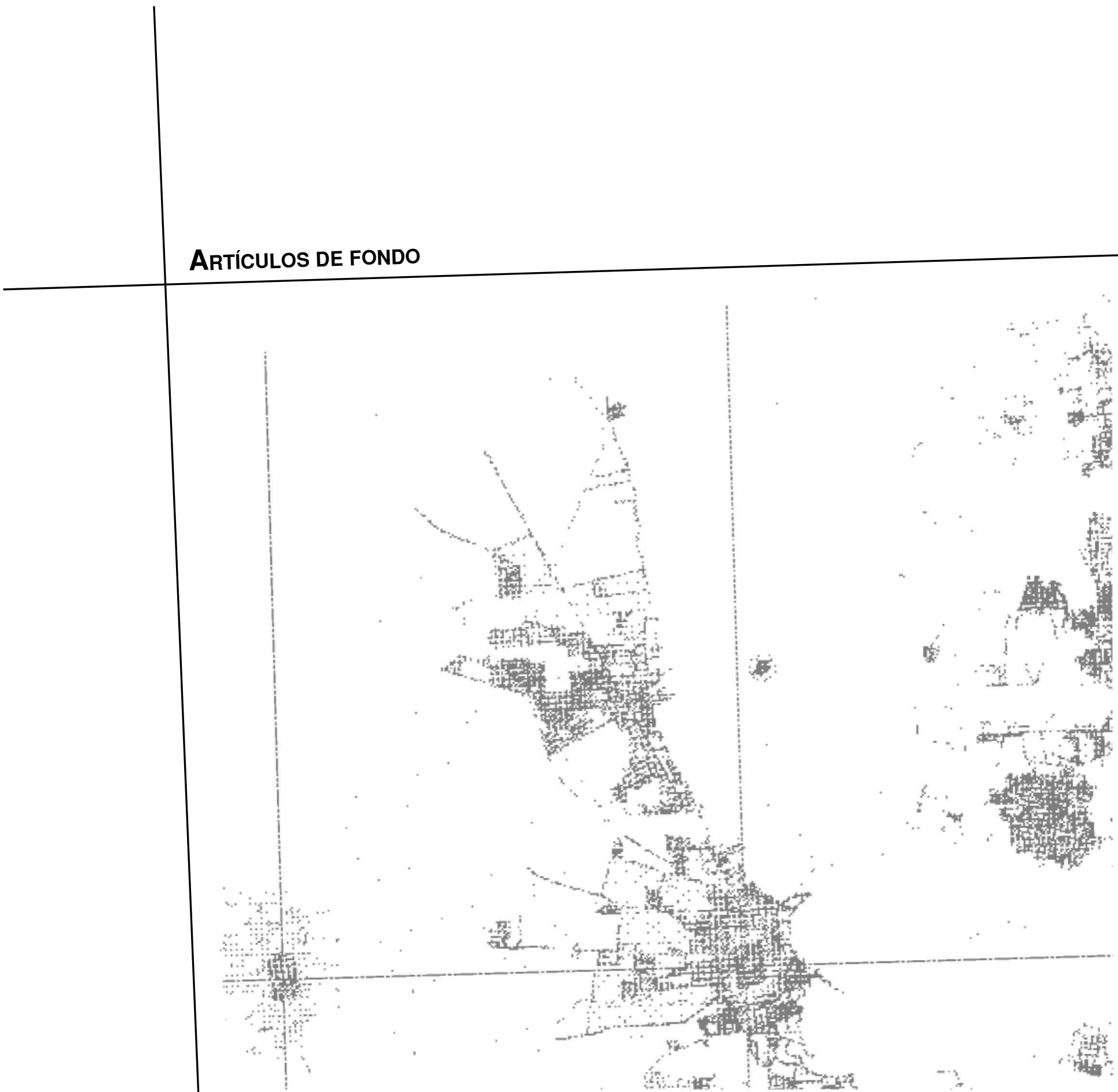





\section{LOS GRANDES PROYECTOS DE DESARROLLO URBANO Y LA RECONFIGURACIÓN SOCIO-ESPACIAL DE LAS CIUDADES: EL BARRIO DE LAVAPIÉS (MADRID)}

\section{Fernando Díaz Orueta}

Doctor en Sociología por la Universidad Complutense de Madrid y técnico urbanista (Instituto Nacional de Administración Pública). Desde 1992 es profesor titular de Sociología Urbana en la Universidad de Alicante. Desde 2002 es miembro del Consejo Directivo del Comité de Investigación 21 (Sociología del Desarrollo Urbano y Regional) de la Asociación Internacional de Sociología (ISA). Dirección postal: Departamento de Sociología II. Universidad de Alicante, Campus de San Vicente del Raspeig. Ap. Correos 99, (03080) Alicante, España. Teléfonos: 0034965903795 / 00 34 965260627.e-mail: fernando.diaz@ua.es

Este artículo fue escrito durante su estancia como Visiting Lecturer en la School of Social Policy, Sociology and Social Research (University of Kent at Canterbury), en el curso académico 2004/2005 (Programa de estancias de profesores de Universidad e investigadores del CSIC y de OPIS en centros de enseñanza superior y de investigación, Ministerio de Educación y Ciencia, Referencia: PR2004-0374) 


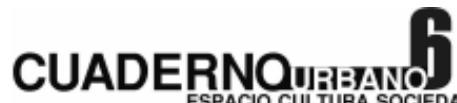

LOS GRANDES PROYECTOS DE DESARROLLO URBANO Y LA RECONFIGURACIÓN SOCIO-ESPACIAL DE LAS CIUDADES: EL BARRIO DE LAVAPIÉS (MADRID)

\section{Resumen}

Madrid es un territorio en transformación. En un corto período de tiempo, tanto la capital como la región han experimentado cambios profundos en sus pautas de organización y crecimiento. Como en otras ciudades, resulta fundamental estudiar su configuración socio-espacial y, particularmente, los procesos de gentrification. En ese sentido, el centro histórico es una de las áreas en las que la reestructuración espacial se percibe con mayor claridad. El artículo se detiene en el estudio de la evolución de Lavapiés, un barrio histórico en el que se desarrolla un importante plan de renovación urbana. La investigación de este espacio urbano permite profundizar en algunas de las claves principales de la reciente evolución socio-espacial de Madrid.

\section{Summary}

Madrid is a territory undergoing transformation. In just a short space of time, the city and the region have seen a modification of well-rooted organisational and reproduction patterns. As in other cities, studying the general socio-spatial configuration and, especially, the processes of gentrification is of utmost importance. The historic centre is one of the areas where the spatial restructuring can be perceived clearly. The article studies the evolution of Lavapiés, a historical neighbourhood where a big urban renovation plan is being developed. Researching about this quarter allow us to deepen on some of the Madrid socio-spatial evolution key patterns. 


\section{Fernando Díaz Orueta}

LOS GRANDES PROYECTOS DE DESARROLLO URBANO Y LA RECONFIGURACIÓN SOCIO-ESPACIAL DE

LAS CIUDADES: EL BARRIO DE LAVAPIÉS (MADRID)

\section{INTRODUCCIÓN}

A lo largo de las dos últimas décadas, una parte sustancial de la investigación urbana ha centrado su atención en el estudio de la reconfiguración socio-espacial de las ciudades. Las transformaciones observadas son de tal calado que permiten referirse a un giro de carácter histórico en la estructuración de los sistemas urbanos. En este artículo se analiza la evolución socio-espacial de Madrid y en particular la de uno de sus barrios centrales: Lavapiés.

Como otras grandes metrópolis europeas, Madrid ha experimentado grandes cambios en los últimos treinta años. En poco tiempo su paisaje se ha visto modificado de forma trascendental, avanzando hacia un modelo de ocupación territorial cada vez más suburbanizado, pero manteniendo una parte importante de la actividad económica en su área central. Tanto el gobierno local como el regional han impulsando diversos proyectos que refuerzan el proceso de reestructuración económica y territorial.

Una de las zonas donde estos cambios resultan más evidentes es el centro de la ciudad y, especialmente, su centro histórico. El artículo se detiene en el estudio de Lavapiés, un barrio que en 1997 fue declarado Área de Rehabilitación Preferente (ARP). Esa declaración dio lugar al impulso de un amplio programa de intervenciones de muy diversa naturaleza. Como en otras áreas de la ciudad, la llegada de población inmigrante extranjera ha transformado rápidamente su estructura social, haciéndola cada vez más heterogénea. Sin embargo, sobre esa realidad social fragmentada se constituyó en 1997 la Red de Colectivos de Lavapiés, entidad que articuló un conjunto de propuestas para el futuro del barrio.

En su primera parte, el artículo recoge el momento actual del debate sobre las transformaciones socio-espaciales en las ciudades, centrándose especialmente en la evolución del concepto de gentrification. Desde su aparición en 1964 el concepto ha ido ganando en complejidad y potencial interpretativo.

A continuación, se realiza una introducción al proceso de reestructuración económica y territorial vivido en Madrid desde los años setenta, cuando sufrió el impacto de la crisis económica. Recientemente, el esfuerzo por convertirse en una ciudad incorporada plena- 


\section{CUADERNOW:and}

172

LOS GRANDES PROYECTOS DE DESARROLLO URBANO Y LA RECONFIGURACIÓN SOCIO-ESPACIAL DE LAS CIUDADES: EL BARRIO DE LAVAPIÉS (MADRID)

1 - Marcuse define la globalización como la combinación de cuatro grandes procesos: a) los avances en la tecnología, especialmente en comunicaciones y transportes; $b$ )

el incremento de la movilidad internacional de personas $y$ capital y del intercambio de mercancías; c) la centralización del control y el aumento de poder de los grandes grupos empresariales respecto al trabajo y a las fuerzas de resistencia, utilizando a y $b$; d) todo ello da lugar a la commodification de bienes, servicios y prácticas y producciones culturales a escala global, maximizando los rendimientos económicos (Marcuse, 2002: 133). El término commodification, traducido al castellano como

“comoditización”, hace referencia a la producción a gran escala de genéricos no diferenciados. mente al sistema urbano europeo se materializaba en los diversos proyectos de transformación amparados bajo el paraguas de la candidatura a los Juegos Olímpicos de 2012.

De este modo, se está en condiciones de contextualizar la evolución de una pieza fundamental de su centro histórico: Lavapiés. En la actualidad, el debate sobre el presente y el futuro del barrio se polariza en dos grandes posiciones. Por un lado, la que entiende que la construcción de grandes equipamientos culturales, las políticas de atracción de nueva población de clase media y el fomento del turismo constituyen la mejor alternativa para este espacio urbano. Por otro, la que ve precisamente en esas actuaciones un riesgo de gentrification, que podría llevar a la expulsión de la población trabajadora, cuya mayoría habita en Lavapiés.

En todo caso, tal y como reflejan las conclusiones, hoy resulta imposible afrontar el análisis del barrio desvinculado de la evolución general de la metrópoli. Su posición central convierte a Lavapiés en un espacio muy afectado por el proceso de reestructuración económico y territorial de Madrid. A través del estudio de un barrio es posible comprender algunas de las claves principales de la reconfiguración global de la ciudad.

\section{REESTRUCTURACIÓN ECONÓMICA Y RECONFIGURACIÓN SOCIO-ESPACIAL DE LAS CIUDADES}

La reestructuración económica iniciada en la década de los setenta resulta un elemento determinante para comprender la naturaleza y profundidad de las transformaciones experimentadas por las ciudades desde entonces. Como plantea Marcuse (2002), el término globalización es utilizado para referirse a un conjunto de acontecimientos de diversa naturaleza que han confluido en estos años y que, como resultado, han provocado un giro de dimensiones históricas. ${ }^{1}$ En esta etapa las políticas urbanas tratan de dotar a cada una de las ciudades de las mejores condiciones para desenvolverse en una red mundial muy jerarquizada y marcada por la competitividad. En la cabecera del sistema urbano se sitúan las ciudades globales, espacios que concentran y coordinan las actividades de comando de la economía mundial (Sassen 1991). 


\section{Fernando Díaz Orueta}

LOS GRANDES PROYECTOS DE DESARROLLO URBANO Y LA RECONFIGURACIÓN SOCIO-ESPACIAL DE LAS CIUDADES: EL BARRIO DE LAVAPIÉS (MADRID)

La creciente homogeneización física y funcional, en numerosas áreas de múltiples ciudades, se ha visto acompañada por un aumento de su fragmentación espacial interna. La proliferación de franquicias, los locales de ocio temático, las obras arquitectónicas de autor, etc., conforman un paisaje urbano homologado, claramente identificable en espacios urbanos muy diversos. La relación arquitectura-administración pública, organizada en torno del impulso de nuevos proyectos cuyo objetivo central es la venta de la ciudad renovada, ha cobrado un enorme vigor. Las intervenciones no se circunscriben a actuaciones aisladas que modifican parcialmente el aspecto de ciertos espacios urbanos. Con frecuencia, se asiste al desarrollo de grandes proyectos que implican la transformación de barrios enteros, con largos tiempos de ejecución e importantes implicaciones sociales. Por tanto, la investigación urbana debe afrontar un escenario complejo de cambios económicos, sociales, políticos y culturales, fuertemente interconectados, y cuyas implicaciones, siendo importantes, van mucho más allá de una redefinición de las pautas de localización residencial de los distintos grupos sociales (Mingione 2004: 68).

En poco tiempo, las ciudades han asistido a la modificación de patrones de organización y reproducción sólidamente asentados. La consolidación de nuevas formas de gobierno y de intervención en el espacio urbano es hoy un hecho constatado que debe ser analizado en cada caso. Estas actuaciones acompañan y refuerzan los cambios en la estructura de clases sociales. Por ejemplo, amplios sectores de clase media han modificado sus patrones de consumo y de localización residencial. La conversión de antiguas zonas industriales y portuarias en declive en nuevas áreas residenciales, de consumo y de ocio, destinadas fundamentalmente a ese grupo social, o la transformación de antiguos barrios de clase trabajadora en lugares de asentamiento de los sectores sociales emergentes son sólo dos muestras de tendencias claramente perceptibles en numerosas urbes.

Generalmente, los grandes proyectos de regeneración urbana se justifican en la necesidad de recuperar un patrimonio arquitectónico degradado y obsoleto. Pero, a la vez, dan respuesta a la necesidad de representación de la nueva economía y a las demandas de nuevo consumo diferenciado: el patrimonio histórico rehabilitado reúne elementos distintivos que aportan prestigio y distinción. En esta categoría no sólo se incluye la rehabilitación de los centros históricos, sino también de espacios industriales abandonados, instalaciones portuarias, viejos mercados de antiguos barrios populares sometidos a procesos 


\section{CUADERNOM:M}

174

LOS GRANDES PROYECTOS DE DESARROLLO URBANO Y LA RECONFIGURACIÓN SOCIO-ESPACIAL DE LAS CIUDADES: EL BARRIO DE LAVAPIÉS (MADRID)

de desinversión, etc. (Lourés 2002). De esta forma, ciertas áreas estratégicas de las ciudades son progresivamente conquistadas y profundamente modificadas, introduciendo nuevas pautas de estructuración socio-espacial. La regeneración urbana se hace visible a través de una serie de ejes fundamentales, entre los que la gentrification ocupa un lugar muy importante.

\section{La gentrification}

En un primer momento, la gentrification fue definida como el proceso a través del cual los habitantes de algunos barrios populares eran desplazados paulatinamente por población de mayores recursos. Glass (1964: 18-19), al analizar la evolución de Londres desde el final de la Segunda Guerra Mundial hasta los inicios de la década de los sesenta, describía el proceso por el cual muchos de los barrios de clase trabajadora fueron ocupados progresivamente por la clase media.

Con el tiempo, la definición fue ganando en complejidad de forma paralela a las grandes transformaciones que tenían lugar en las ciudades. Desde la década de los ochenta, estos procesos aumentaron notablemente, adquiriendo características propias, en función de los contextos sociales, políticos y económicos en los que se producían. El estudio de Zukin (1982) sobre el Soho neoyorquino tuvo una gran importancia en la discusión sobre el sentido de la gentrification, destacando cómo la población de mayores ingresos genera una demanda de bienes y servicios que, frecuentemente, son producidos de forma no masiva y vendidos a través de establecimientos al por menor.

En este sentido, Hamnett (2003) enfatiza la importancia de la expansión de la clase media urbana en la profundización de la gentrification a partir de los años ochenta. La reestructuración económica ha hecho crecer el peso de grupos profesionales con demandas de consumo, culturales, residenciales, etc., diferenciadas. Progresivamente, una fracción amplia de estos sectores emergentes ha ido ocupando determinadas zonas de las ciudades (especialmente algunas áreas centrales), transformándolas no sólo físicamente (rehabilitación de viviendas, edificios, construcción de nuevos equipamientos y servicios, etc.), sino también socialmente, con nuevos estilos de consumo, de reproducción social (educación 


\section{Fernando Díaz Orueta}

LOS GRANDES PROYECTOS DE DESARROLLO URBANO Y LA RECONFIGURACIÓN SOCIO-ESPACIAL DE LAS CIUDADES: EL BARRIO DE LAVAPIÉS (MADRID)

y socialización), etc. Ahora bien, como plantean Butler y Robson (2003) al referirse a Londres, muchos de estos barrios son lugares premeditadamente aislados del resto de la ciudad, empeñados en una búsqueda de la distinción (Bourdieu 1991) y la exclusividad.

A partir de la revisión de una serie de investigaciones y estudios de caso, Carpenter y Lees (1995) sistematizaron las distintas etapas que se pueden observar en un proceso de gentrification. De manera esquemática los pasos son: a) la suburbanización o, en el contexto europeo continental, el desplazamiento de la población hacia las periferias urbanas; b) la desinversión, con el consiguiente abandono físico del barrio; c) el abandono de las viviendas por parte de los propietarios privados; d) el paso de la desinversión a la inversión. En este momento surge el cambio de tendencia que, en ocasiones, coincide con el anuncio del impulso de un plan de intervención; e) la reinversión a gran escala, con agentes públicos y privados; f) la financiación por parte del Estado; g) el desarrollo de las formas de consumo ligadas a la gentrification.

Gottdiener y Budd (2005) remarcan que la gentrification camina ligada al declive de ciertos espacios urbanos. Como Carpenter y Lees (1995), también ellos consideran clave el proceso de desinversión que, irremediablemente, lleva al abandono. El declive puede producirse de forma más o menos inducida. La presencia de población de escasos recursos en ciertos barrios de la ciudad es percibida por inversionistas del sector inmobiliario como un impedimento para obtener beneficios económicos. En ese sentido, la actitud que tomen los gobiernos locales resulta fundamental, favoreciendo los intereses de estos sectores o, por el contrario, impulsando intervenciones que traten de frenar el proceso de gentrification. Cuando se produce la confluencia entre el sector público y el sector privado para favorecer el desplazamiento de los sectores populares, es muy frecuente la utilización del miedo y la inseguridad para legitimar socialmente estas operaciones. Como destaca Kesteloot (2004: 142), este uso del miedo es todavía más potente si, además, la población a expulsar es inmigrante:

Cuando de una forma u otra el abandono y el declive se han instalado en un barrio, ¿cómo se revierte la tendencia y se comienza a atraer el capital que favorecerá el proceso de renovación?, iqué causas provocan este cambio de tendencia? De acuerdo con las experien- 


\section{CUADERNOM:M}

176

LOS GRANDES PROYECTOS DE DESARROLLO URBANO Y LA RECONFIGURACIÓN SOCIO-ESPACIAL DE LAS CIUDADES: EL BARRIO DE LAVAPIÉS (MADRID)

2- Sassen (2003: 50) se refiere al retorno de las llamadas clases de servidumbre, formadas ahora por inmigrantes y mujeres. cias conocidas, la puesta en práctica de un plan público de actuación puede jugar un papel importante al suponer una renovación de infraestructuras, equipamientos, etc., por cuenta de las arcas públicas. Además, con este tipo de intervenciones, la Administración envía una señal de confianza a los inversores privados que pasarán a ver la zona como un área atractiva (Gottdiener y Budd 2005: 33)

En ocasiones, el paso desde el declive a la reinversión se produce sin que medie un plan de intervención a gran escala. En gran medida, la evolución reciente del barrio de Chueca (Madrid) respondería a este modelo. Un espacio urbano en declive ha sido renovado de la mano del asentamiento de una numerosa comunidad gay, económicamente muy solvente, que ha convertido el barrio en una referencia internacional de este colectivo. A diferencia de lo ocurrido en otras áreas del centro histórico de Madrid, no ha existido ningún plan especial de actuación.

A la vez, la gentrification descansa sobre la disponibilidad de amplios sectores de trabajadores con salarios bajos. De hecho, una parte significativa del empleo creado en los últimos años se concentra en actividades para las que se requiere una escasa calificación profesional y en las que predomina la precariedad laboral: la construcción, la limpieza, la hostelería, la mensajería, etc. Como plantea Sassen (2003:50), existe una relación directa entre las necesidades de las clases medias profesionales y gerenciales de las ciudades globales y el crecimiento de los empleos precarios. Los estilos de vida tradicionales resultan incompatibles con las necesidades que afrontan cotidianamente estos sectores emergentes. Este desajuste es cubierto en proporciones importantes por población inmigrante que se incorpora al mercado laboral en condiciones muy desfavorables. ${ }^{2}$ Se alimenta así un crecimiento de la desigualdad que se expresa en la reproducción simultánea de espacialidades diferentes, protagonizadas por los diversos grupos sociales que habitan las ciudades (Sassen 1991).

\section{MADRID: EL PROYECTO DE CIUDAD GLOBAL}

La segunda mitad de los años ochenta fue un tiempo de fuerte crecimiento económico. Las inversiones extranjeras se dispararon de manera exponencial en España, siendo Ma- 


\section{Fernando Díaz Orueta}

LOS GRANDES PROYECTOS DE DESARROLLO URBANO Y LA RECONFIGURACIÓN SOCIO-ESPACIAL DE LAS CIUDADES: EL BARRIO DE LAVAPIÉS (MADRID)

drid la principal receptora. A partir del momento de la incorporación a la entonces conocida como Comunidad Económica Europea (CEE), las ayudas económicas de ese continente fluyeron con generosidad. La crisis parecía haber sido superada. El desempleo se redujo, aunque sobre la base de la extensión de la precariedad. De hecho, cuando a partir de 1992 el crecimiento económico se volvió a estancar, la destrucción de empleo fue muy acelerada, con un rápido crecimiento de las tasas de paro. Más adelante, en la nueva fase expansiva, la Comunidad de Madrid creó empleo a un ritmo más intenso que la media española: entre 1995 y 2004 un 42\%, alcanzándose en este último año los 2,4 millones de trabajadores ocupados (Encuesta de Población Activa).

De la intensidad de la etapa de crecimiento vivido estos años da cuenta la fuerte corriente migratoria, que ha convertido a la Comunidad de Madrid en la región española con mayor presencia de extranjeros. Desde los años ochenta se comenzó a detectar una nueva corriente de inmigración (IOE 1991), que aumentó notablemente en los noventa hasta convertirse en un flujo muy importante con una composición heterogénea. A principios de 2005 los inmigrantes extranjeros suponían el 15,2\% de la población de la ciudad (Padrón Municipal de Habitantes). Así, y fundamentalmente debido a este movimiento de la población, la tendencia a la pérdida demográfica se ha visto rota en el último quinquenio: la ciudad de Madrid contaba en 1999 con 2.879 .052 habitantes que habían pasado a ser 3.092.759 en 2003. Este hecho supone una auténtica novedad histórica. Debe recordarse que los grandes movimientos migratorios de los años sesenta fueron protagonizados por españoles procedentes de otras zonas del país. Ahora Madrid ha visto asentarse en muy pocos años a cientos de miles de extranjeros procedentes de todos los rincones del planeta. Sus pautas de localización residencial se aproximan a las de la población autóctona de parecidas características socioeconómicas, compartiendo hábitat con las clases trabajadoras (Labrador y Merino 2002). Debe resaltarse su presencia en un número significativo en ciertas áreas del centro de la ciudad, aquellas en las que existe una oferta de vivienda a precio más asequible.

El ayuntamiento de Madrid y el gobierno autonómico han acompañado y favorecido el proceso de reestructuración. Desde la política urbana y territorial se han ido poniendo en práctica las medidas que podían consolidar a Madrid como una ciudad incorporada al 


\section{CUADERNOW:and}

LOS GRANDES PROYECTOS DE DESARROLLO URBANO Y LA RECONFIGURACIÓN SOCIO-ESPACIAL DE LAS CIUDADES: EL BARRIO DE LAVAPIÉS (MADRID)

3 -De acuerdo con un estudio del Instituto Geográfico Nacional, entre 1990 y 2000 la superficie construida en la Comunidad Autónoma de Madrid aumentó en un 49,2\%. En esos años la población se incrementó en una cuantía mucho menor: un 9,6\% (Méndez 2004: 28-29).

4- Junto a una consolidación de la ciudad y de la región como destino turístico de carácter

cultural. En esa clave deben ser entendidas, por ejemplo, las ampliaciones del Museo del

Prado, del Museo Thyssen o del Museo de Arte Reina Sofía, todos ellos situados en el conocido Paseo de las Artes de Madrid. sistema urbano europeo, aunque en un segundo escalón respecto de las principales ciudades globales. En los discursos de los políticos madrileños aparece constantemente la referencia a la globalización y a la competitividad. Ya en marzo de 1991 el entonces alcalde de la ciudad, Agustín Rodríguez Sahagún, se manifestaba de la siguiente forma al presentar una exposición sobre el Plan Estratégico de Madrid:

"A partir de 1993, Madrid tiene el reto de convertirse en una ciudad más competitiva en sus áreas productivas y más habitable para sus residentes de lo que es en la actualidad. Nuestra ciudad, para alcanzar un rango similar al de otras capitales europeas, tiene que asumir un proyecto innovador que le permita afrontar con éxito los riesgos previsibles y aprovechar inteligentemente las oportunidades de futuro."

A lo largo de estos años se ha observado una actuación coordinada entre los distintos equipos de gobierno municipal y regional con los grandes grupos empresariales, las Cámaras de Comercio, los principales medios de comunicación, etc., constituyendo una poderosa coalición por el crecimiento (Logan y Molotch 1987). En ese contexto, debe ser entendido el esfuerzo de inversión en grandes infraestructuras (transporte, telecomunicaciones, etc.), que ha respaldado el proceso de reestructuración económica. La intensificación del proceso urbanizador ha sido espectacular, la ampliación y extensión de la red de carreteras y autopistas y de los ferrocarriles de cercanías han favorecido una auténtica explosión de la mancha urbana. En oleadas sucesivas, se ha producido el crecimiento de municipios metropolitanos situados en coronas cada vez más alejadas del núcleo central, incorporando localidades extra-metropolitanas hasta hace escasos años. En un corto período de tiempo, el territorio regional se ha transformado drásticamente. ${ }^{3}$

Las modalidades de actuación de los gobiernos locales y del gobierno regional se han alineado con lo observado en otras ciudades europeas. El impulso de grandes proyectos urbanos en la capital, tendencia todavía más evidente tras la candidatura de Madrid para los Juegos Olímpicos de 2012, se ha visto reforzado por intervenciones de alcance más limitado en ciertos barrios de la ciudad o de otros municipios de la Corona Metropolitana. La ampliación del Aeropuerto de Barajas, la construcción del parque temático de Warner en San Martín de la Vega y el desarrollo de nuevos centros comerciales y de ocio, repartidos por toda la región, son sólo algunas otras muestras de esta gran diversidad de actuaciones. ${ }^{4}$ 


\section{Fernando Díaz Orueta}

LOS GRANDES PROYECTOS DE DESARROLLO URBANO Y LA RECONFIGURACIÓN SOCIO-ESPACIAL DE

LAS CIUDADES: EL BARRIO DE LAVAPIÉS (MADRID)

\section{Los procesos de diferenciación socio-espacial y la vivienda}

Uno de los principales problemas que ha debido afrontar la sociedad madrileña como consecuencia de la orientación dada al modelo económico-territorial, es el acceso a la vivienda. Ya durante la segunda mitad de los ochenta el crecimiento de los precios y la debilidad de las políticas de vivienda generaron una enorme bolsa de ciudadanos para los que la vivienda se convirtió prácticamente en un bien inalcanzable (Leal 1987). En 1997 se inició una nueva fase de crecimiento pronunciado de los precios. En la actualidad Madrid es una de las capitales europeas donde el acceso a la vivienda resulta más complicado. Como afirma Roch, esto ocurre en un territorio con un crecimiento demográfico moderado, con una enorme existencia de viviendas disponibles:

"Sólo en la metrópoli madrileña, donde existen más de 300.000 viviendas vacías y otras 275.000 claramente infrautilizadas, se vienen superando las 40.000 nuevas viviendas en los últimos años y hay una disponibilidad de suelo ya clasificado para otras 800.000 viviendas, que permitirían alojar holgadamente a Valencia y Sevilla juntas" (Roch 2004: 32).

Roch liga esta situación al desarrollo de un nuevo espacio social en Madrid, caracterizado por la jerarquización en áreas de composición socioeconómica cada vez más homogénea y profundamente marcada por los precios de la vivienda. Esta situación tiene lugar en un contexto caracterizado por la alianza estratégica entre el capital financiero y los agentes inmobiliarios. La ciudad se convierte así en un espacio de acumulación de rentas familiares: a través de las viviendas construidas el ahorro se materializa en patrimonio inmobiliario y familiar. Pero este modelo, además de ser enormemente destructivo desde el punto de vista medioambiental, genera una gran segregación espacial (Roch 2003: 117). De hecho, algunos estudios comparativos han confirmado una mayor tendencia a la segregación en Madrid que en otras ciudades europeas, ligándolo al desarrollo particular de su modelo inmobiliario. Preteceille (2000: 87), en línea con lo mantenido por Leal, plantea como hipótesis explicativas el escaso alcance de las políticas públicas de vivienda y la opción masiva por la vivienda en propiedad.

Segregación que, como ya se adelantaba, se ha visto acompañada de un gran crecimiento en la Comunidad de Madrid. Entre 1981 y 2001, la región pasó de 4.687 .523 a 5.372 .433 


\section{CUADERNOW:Band}

180

LOS GRANDES PROYECTOS DE DESARROLLO URBANO Y LA RECONFIGURACIÓN SOCIO-ESPACIAL DE LAS CIUDADES: EL BARRIO DE LAVAPIÉS (MADRID)

5 - Sobre la evolución espacial de la vivienda en la Comunidad de Madrid: Leal y Cortes, 1995.

6- Respecto al Plan General de 1985, su filosofía y sus resultados: Ateneo Madrileñista, 1989. habitantes (censos de población de 1981 y 2001). Durante este período se ha asistido a un proceso de creciente suburbanización: a) hacia el sur y el este, alimentado mayoritariamente por la necesidad de encontrar una vivienda a precio asequible, y b) hacia las urbanizaciones del norte y el oeste, en un movimiento predominantemente protagonizado por la clase media. Por supuesto, estas son tendencias muy generales que deben matizarse en el análisis detallado de distintas áreas. ${ }^{5}$ Los grandes ejes de diferenciación espacial se refuerzan sobre sus raíces históricas y, simultáneamente, adquieren nuevas formas y así, entendida globalmente, Madrid dista mucho de poder ser caracterizada exclusivamente en clave de una dualización socioeconómica. En realidad, su estructura socio-espacial respondía, ya a principios de los años noventa, a la hipótesis de la ciudad fragmentada, tendencia reforzada recientemente por la evolución del mercado inmobiliario (Díaz Orueta 2001).

\section{Intervenciones urbanas en el centro histórico de Madrid}

Después de más de cuatro décadas, en 1979 se celebraban elecciones municipales en España. En Madrid, tras el acuerdo de gobierno alcanzado por el PSOE y el PCE, Enrique Tierno Galván se convertía en alcalde de la ciudad. El nuevo Plan General de Madrid fue aprobado en 1985 y se inspiró en gran medida en la experiencia del gobierno de la izquierda en distintas ciudades italianas, recogiendo los principios del llamado Urbanismo de la Austeridad. Ideas como la de romper las desigualdades entre el norte y el sur de Madrid, el derecho a la ciudad para todos, el cierre de las heridas en la ciudad existente evitando fomentar grandes crecimientos, inspiraron este documento. ${ }^{6}$ En ese contexto, debe entenderse una preocupación específica por el centro histórico, muy abandonado hasta ese momento y sometido a un fuerte proceso de terciarización y envejecimiento de la población. Así, la recuperación de su función residencial y el mantenimiento de sus habitantes tradicionales fueron dos objetivos primordiales. De hecho, el nuevo Plan General asumió el Plan Especial de Protección y Conservación de edificios y conjuntos de carácter histórico-artístico en la Villa de Madrid, más conocido como Plan Especial Villa de Madrid, el cual había sido aprobado en octubre de 1980, y catalogó más de 2.500 edificios tanto del casco antiguo como del ensanche y de los doce cascos de pueblos absorbidos por la ciudad en su crecimiento, distinguiendo tres niveles de protección (integral, estructural y ambiental). El principal objetivo fue dar respuesta a la situación creada en 


\section{Fernando Díaz Orueta}

LOS GRANDES PROYECTOS DE DESARROLLO URBANO Y LA RECONFIGURACIÓN SOCIO-ESPACIAL DE LAS CIUDADES: EL BARRIO DE LAVAPIÉS (MADRID)

décadas anteriores, promoviendo la rehabilitación del enorme patrimonio arquitectónico existente y fomentando el uso residencial.

Globalmente sus resultados pueden considerarse ambivalentes: por un lado, se frenó a la destrucción patrimonial que en años anteriores avanzaba implacable sobre muchas áreas del centro. Pero las medidas puestas en práctica no fueron suficientes para promover una rehabilitación masiva que alcanzase los objetivos antes descritos. Años más tarde, en 1997, el nuevo Plan General de Madrid cambió por completo su filosofía. En un escenario económico y político muy diferente, se apostó por el crecimiento urbano a gran escala, la creación de nuevas infraestructuras viarias de gran capacidad y, desde el punto de vista patrimonial, por la descatalogación de edificios. El discurso de la austeridad había quedado atrás.

\section{LAS ÁREAS DE REHABILITACIÓN PREFERENTE (ARP): LAVAPIÉS}

En 1994 las administraciones local, autonómica y central firmaron un convenio de colaboración cuyo propósito era impulsar la rehabilitación del casco antiguo de Madrid y de los barrios periféricos degradados. Para ello se contemplaba una serie de figuras de intervención entre las que destacaban las llamadas ARP. Sus objetivos eran: mejoramiento de la calidad de vida, recuperación del carácter residencial, regeneración del tejido social, mejora de las viviendas y edificios, impulso del crecimiento económico, creación de nuevas dotaciones y espacios públicos y promoción de la participación ciudadana (Empresa Municipal de la Vivienda 1999). Como señala Lourés (2003), la propuesta debe ser entendida en el contexto del impulso que en aquellos momentos se daba en diversos países europeos a este tipo de figuras de actuación. Asimismo, formaba parte de una estrategia más amplia destinada a "sanear" el centro de la ciudad, convirtiéndolo en un espacio apto para el turismo.

Cuando en 1997 fue aprobado el Plan General, se incorporaron a él todas las ARP declaradas hasta ese momento. El mismo año de aprobación del Plan se puso en marcha también el ARP de Lavapiés. El área sobre la que se intervino, una parte muy amplia del barrio, se denominó Sector I. Se trataba de una superficie de 34,5 hectáreas, con 766 edificios, 11.878 


\section{CUADERNOM:}

182

LOS GRANDES PROYECTOS DE DESARROLLO URBANO Y LA RECONFIGURACIÓN SOCIO-ESPACIAL DE LAS CIUDADES: EL BARRIO DE LAVAPIÉS (MADRID)

7- Embajadores, junto con los barrios de Palacio, Cortés, Justicia, Universidad y Sol, componen el distrito Centro de la ciudad. viviendas y una población de 19.706 habitantes. 4.969 viviendas fueron calificadas como infraviviendas. Una ampliación posterior - julio de 2003- extendió la zona de actuación. De acuerdo con la normativa de las ARP, la naturaleza de la actuación planteada era claramente multidimensional: la renovación de las infraestructuras (luz, alcantarillado, agua, gas, etc.), la subvención a la rehabilitación privada de viviendas, el desarrollo de programas de adecuación arquitectónica, la puesta en marcha de un plan de intervención social o la creación de nuevos equipamientos. Asimismo, una acción paralela a las recogidas en el plan para Lavapiés fue el denominado Proyecto Medioambiental, financiado por los fondos de cohesión europeos.

Lavapiés forma parte del distrito centro de la ciudad de Madrid, localizándose geográficamente en su zona sur. Sus comunicaciones son excelentes, diversas líneas de metro, autobús y ferrocarril rodean el barrio, desde el que además se puede acceder fácilmente a pie a una gran parte del centro de la ciudad. Aunque administrativamente se incluye en un barrio mayor (Embajadores), ${ }^{7}$ sin embargo presenta una personalidad claramente diferenciada. Lavapiés tradicionalmente ha sido un espacio de clase trabajadora conservando, en contraste con otros espacios del centro muy terciarizados, un gran dinamismo social muy identificado con el casticismo madrileño (Lourés 2003: 96).

Tímidamente primero y de forma masiva más adelante, Lavapiés pasó a ser uno de los destinos elegidos por la nueva población inmigrante llegada a Madrid. La existencia de una oferta de vivienda en alquiler mayor que en otras áreas de la ciudad y unos precios claramente menores atrajeron a estos ciudadanos. Propietarios sin muchos escrúpulos han aprovechado la situación para conseguir pingües beneficios, permitiendo la acumulación de un gran número de personas en pisos reducidos y de pésima calidad o, incluso, utilizando el sistema de "camas calientes", que significa el alquiler individualizado por horas de cada una de las camas instaladas en una vivienda.

En la segunda mitad de los años noventa, y a pesar de que muchos inmigrantes no disponían de papeles, su llegada se reflejaba ya claramente en las estadísticas municipales. Si en 1996, habitaban en el barrio 28.418 habitantes, en 2000 esa cantidad había ascendido hasta los 29.662 (EDIS 2000). Una evolución semejante mantenía no sólo el barrio de Embajadores al completo (de 40.475 a 42.573), sino incluso el distrito centro en su conjunto 


\section{Fernando Díaz Orueta}

LOS GRANDES PROYECTOS DE DESARROLLO URBANO Y LA RECONFIGURACIÓN SOCIO-ESPACIAL DE LAS CIUDADES: EL BARRIO DE LAVAPIÉS (MADRID)

(de 122.615 a 130.473). Se rompía así con una pérdida de población que se había mantenido interrumpidamente durante varias décadas. La recuperación demográfica se ha visto acompañada, además, del rejuvenecimiento de la estructura de edades, con una presencia creciente de los grupos infantil y juvenil. De acuerdo con las estadísticas municipales recogidas en el informe de EDIS, en el año 2000 residían 3.865 extranjeros en el barrio, de los cuales un 13,1\% estaba conformado por menores de 15 años y un 30,5\% tenía entre 15 y 29. De hecho, los colegios públicos del barrio afrontan una situación compleja, derivada de la llegada masiva y no esperada de nuevos estudiantes, muchos de los cuales desconocen la lengua española.

En los primeros años del siglo XXI estas tendencias se han acentuado todavía más. Datos actualizados del Padrón de Habitantes a l de enero de 2005, dan cuenta de cómo el 39,5\% de toda la población en edad escolar (menores de 16 años) del barrio de Embajadores es extranjero: 2.173 estudiantes sobre un total de 5.499. Entre el 1 de enero de $2004 \mathrm{y}$ el 1 de enero de 2005, el número de españoles en el barrio habría disminuido en 261, mientras que el de extranjeros se habría visto incrementado en 1.531. Embajadores contaba ya en esa fecha con 52.268 habitantes.

Un rasgo muy importante de la población inmigrante del barrio es la diversidad de sus procedencias. Ecuatorianos y marroquíes son las comunidades más numerosas, pero existe una presencia muy significativa de otras nacionalidades (Lourés 2003). Para percibir la enorme diversidad cultural del barrio basta con darse un paseo por su plaza principal: la Plaza de Lavapiés. La estructura comercial se ha visto también muy transformada, con la aparición de nuevos establecimientos ligados con la inmigración (locutorios, peluquerías, carnicerías, etc.).

Otros grupos han aparecido en el barrio, profundizando su heterogeneidad. En primer lugar, la presencia del movimiento okupa se mantiene ininterrumpidamente desde la década de los ochenta. Protagonizado mayoritariamente por jóvenes procedentes de diversas zonas de Madrid, han ocupado diversos edificios del barrio, llevando adelante actividades sociales y culturales, a la vez que denuncian el carácter especulativo del modelo urbanístico actual. La okupación que ha contado con un mayor impacto fue la llevada a cabo en 1997 en el antiguo edificio del Instituto de Investigaciones Agrarias. Conocida como El 


\section{CUADERNOM:M}

184

LOS GRANDES PROYECTOS DE DESARROLLO URBANO Y LA RECONFIGURACIÓN SOCIO-ESPACIAL DE LAS CIUDADES: EL BARRIO DE LAVAPIÉS (MADRID)

Laboratorio (El Labo), pronto pasó a ser un importante foco de actividad asociativa, abierto a la utilización de otros grupos. Sucesivos desalojos fueron seguidos de la okupación de nuevos edificios, hasta llegar a la cuarta y última expulsión, por el momento.

En segundo lugar, un grupo muy variado de distintos profesionales y artistas relacionados con el mundo de la música, el teatro, etc., han elegido el barrio como su lugar de residencia. Estas personas dicen sentirse atraídas precisamente por la diversidad cultural del lugar, su posición central en la ciudad, su carácter histórico, su ambiente nocturno, etc. En gran medida responderían al perfil de lo que algunos autores (Simon 2004: 220223) han denominado como multiculturales. Personas con un alto nivel educativo y una gran capacidad de consumo que trabajan en profesiones liberales, artísticas, en la educación, etc. En Lavapiés mantienen mayoritariamente una actitud de compromiso con el barrio apoyando sus movilizaciones.

Aunque cuantitativamente el volumen de estos sectores es limitado, su presencia resulta muy importante en la medida que contrarresta parcialmente la estigmatización sufrida por Lavapiés en los últimos años. Desde diferentes ámbitos políticos, respaldados por algunos medios de comunicación, se ha presentado el barrio como un lugar peligroso ocupado por grupos de delincuentes que lo habrían convertido en un "territorio sin ley". Se ha atacado también la presencia del movimiento okupa, ligándolo incluso a grupos violentos que utilizarían Lavapiés como plataforma de sus actividades. Esta visión se vio muy reforzada tras los atentados terroristas del 11 de marzo de 2004, aprovechando la vinculación con el barrio de alguno de los implicados. En definitiva, se intenta convencer a la población de Madrid de que Lavapiés se ha convertido en un gueto peligroso en el corazón de la urbe y que, por tanto, urgiría llevar adelante una "limpieza profunda".

\section{Actuaciones urbanas en el área}

El monto total de la inversión pública comprometida ha sido muy importante. En los primeros años se llevó a cabo una profunda renovación de las infraestructuras (canalizaciones de agua, gas, alcantarillado, etc.). Asimismo, se procedió a reordenar el tráfico en la zona, con un nuevo tratamiento de las calzadas y la creación de aparcamientos subterráneos. En este sentido, la principal operación fue la creación de un gran aparcamiento 


\section{Fernando Díaz Orueta}

LOS GRANDES PROYECTOS DE DESARROLLO URBANO Y LA RECONFIGURACIÓN SOCIO-ESPACIAL DE LAS CIUDADES: EL BARRIO DE LAVAPIÉS (MADRID)

subterráneo en la Plaza de Agustín Lara, uno de los mayores espacios abiertos del barrio. Esta actuación fue acompañada de una remodelación global de la plaza. La renovación de las infraestructuras ha sido una operación valorada positivamente en el barrio, aunque en su momento se produjera un gran descontento por la lentitud de unas obras que, por varios años, llenaron el barrio de barreras. Distintas asociaciones han criticado el deterioro acelerado de muchas de estas obras debido, básicamente, a la falta de mantenimiento.

Otro campo en el que se percibe con claridad la intervención en el barrio es en el desarrollo de nuevos equipamientos. La construcción de un nuevo Centro Asociado y una Biblioteca de la Universidad Nacional de Educación a Distancia (UNED), la nueva Sala del Instituto Nacional de Artes Escénicas y de la Música (INAEM) levantada en el solar que anteriormente ocupaba la Sala Olimpia, la rehabilitación para centro social del antiguo Casino de la Reina y de sus jardines, son algunas de las operaciones ya ejecutadas. Asimismo, y de manera paralela al plan, la Caja de Madrid llevó adelante la rehabilitación de un antiguo edificio neomudejar, convirtiéndolo en centro cultural (la Casa Encendida). Todas ellas, junto con los planes previstos para la antigua Fábrica de Tabaco en la que la administración planea la instalación de varias salas de exposición y de dos nuevos museos, dan cuenta de una estrategia planteada en clave global, orientada a satisfacer prioritariamente los consumos de la clase media y el desarrollo turístico de la ciudad. Antiguas reivindicaciones barriales como la construcción de un nuevo centro de salud o la mejora de los colegios públicos, desbordados por la llegada de nuevos alumnos, han sido ignoradas hasta la fecha.

En cuanto a la rehabilitación de viviendas, eje fundamental del plan, su alcance ha quedado por debajo de las expectativas generadas inicialmente. La operación se ha dirigido, fundamentalmente, a la subvención a los propietarios. Sin embargo, dado que no se produjo una valoración correcta de las condiciones socioeconómicas de la población residente, ni del régimen de explotación extrema que muchos propietarios aplican a sus viviendas, las peticiones de ayuda han quedado muy por debajo de lo planeado inicialmente y circunscritas, sobre todo, a aquellos vecinos con una mayor capacidad económica. Asimismo, la gestión de las ayudas ha sido extremadamente lenta y compleja. Entretanto, el precio de la vivienda ha continuado creciendo, aunque todavía mantenga un diferencial significativo con otras áreas del centro de la ciudad. Numerosos propietarios 


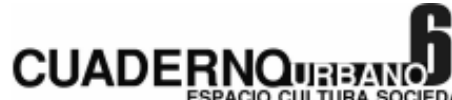

186

LOS GRANDES PROYECTOS DE DESARROLLO URBANO Y LA RECONFIGURACIÓN SOCIO-ESPACIAL DE LAS CIUDADES: EL BARRIO DE LAVAPIÉS (MADRID)

incumplen su obligación de mantener los edificios, esperando una declaración de ruina que permita el derribo y la construcción de nuevas viviendas. Esta práctica se mantiene de forma sostenida, tanto en Lavapiés, como en otras áreas del centro histórico de Madrid, favorecida por la descatalogación de edificios incluida en el Plan General de 1997.

En 2004 el Ayuntamiento de Madrid aprobó un nuevo plan para el centro de la ciudad. Denominado Plan de Acción de Urbanismo, Vivienda e Infraestructuras para la revitalización del Centro Urbano (PERCU), en él se sistematizan distintas actuaciones y se proponen algunas nuevas, en el marco de las operaciones urbanas previstas ante la candidatura finalmente fracasada de Madrid para las Olimpíadas de 2012. Lavapiés se ve afectada con una nueva ampliación de la zona incluida en el ARP. Con esta tercera fase, a desarrollar entre 2006 y 2008, se cubre el conjunto del espacio barrial, alcanzándose las 70,82 hectáreas y las 22.278 viviendas.

\section{La participación ciudadana}

La normativa sobre las ARP considera uno de sus objetivos incentivar la participación ciudadana. Sin embargo, en Lavapiés la intervención ha avanzando sin que la Administración implementase canales que la propicien. Los habitantes de Lavapiés tuvieron noticia de la intervención como algo decidido y cerrado en sus detalles, sin que en ningún momento hubieran podido hacer llegar sus opiniones y expectativas. Por un lado, desde el tejido social del barrio se celebró haber alcanzado una reivindicación, la rehabilitación, que venía siendo planteada desde los años setenta por la Asociación de Vecinos La Corrala. Por otro, se extendió todo tipo de sospechas sobre cuáles podrían ser los objetivos no manifiestos de esa intervención.

Ante este anuncio, una serie de grupos y organizaciones del barrio, coordinados por la Asociación de Vecinos la Corrala, intensificó sus contactos. El planteamiento central fue la defensa de la rehabilitación del barrio, pero planteada desde una perspectiva que colocaba en primer plano las necesidades de sus habitantes actuales. Nació así en octubre de 1997 la Red de Colectivos de Lavapiés, compuesta por una amplísima variedad de asociaciones y grupos: el viejo asociacionismo barrial representado por La Corrala, que simboli- 


\section{Fernando Díaz Orueta}

LOS GRANDES PROYECTOS DE DESARROLLO URBANO Y LA RECONFIGURACIÓN SOCIO-ESPACIAL DE

LAS CIUDADES: EL BARRIO DE LAVAPIÉS (MADRID)

zaba la continuidad con anteriores etapas de movilización, las nuevas asociaciones articuladoras de los distintos colectivos de inmigrantes (marroquíes, senegaleses, guineanos, etc), diversas expresiones del movimiento okupa, ONG (Xenofilia, Paideia, SOS Racismo, etc), grupos culturales, etc. La Red se consolidó como un espacio de encuentro que, con vaivenes, ${ }^{8}$ se ha mantenido hasta la fecha.

Desde el principio, la Red, junto con otros grupos del barrio, planteó como uno de sus objetivos centrales la necesidad de utilizar como centro social algunos edificios en desuso y de titularidad pública, donde pudieran realizarse las diversas actividades planteadas por los grupos que la componían. Esa propuesta llegó a un alto nivel de concreción, cuando en febrero de 1999 la Red presentó ante el Ayuntamiento de Madrid el llamado Proyecto para Equipamientos Sociales, en el que se especificaban no sólo las actividades a desarrollar sino los lugares propuestos dentro del barrio. La Administración accedió a regañadientes al diálogo con la Red, aprobando en diciembre de 2000 el llamado Programa de Intervención Social que subvencionó los proyectos de varios grupos. Sin embargo, no se admitió la propuesta de utilización de diversos edificios.

La Red, además de otras organizaciones, ha participado y propiciado a partir de 2003 la discusión sobre los futuros usos que le darían al edificio del siglo XVIII de la antigua Fábrica de Tabacos. Desde hace años esta propiedad del Ministerio de Cultura, de más de 28.000 metros cuadrados, no es utilizada. Se trata de uno de los edificios cuyo aprovechamiento fue planteado por la Red para albergar las actividades de su propuesta de recuperación de espacios públicos para Equipamientos Sociales. Sin embargo, aunque hasta la fecha no se ha tomado todavía una decisión definitiva, el Ministerio parece considerar la apertura de dos salas de exposiciones temporales y la posible apertura del Museo $\mathrm{Na}$ cional de Artes Decorativas y del Museo de Reproducciones Artísticas. La Red solicita que al menos se les ceda una parte de las instalaciones para desarrollar sus actividades.

Tanto la Red de Lavapiés como la Red La Tabacalera a Debate vinculan esta operación y, en general el conjunto de las actuaciones emprendidas en Lavapiés, con la evolución seguida por Madrid en los años más recientes, criticando un modelo de ciudad sostenido por la candidatura para las Olimpíadas de 2012 y el nuevo Plan PERCU:
8- A lo largo de estos años la Red ha realizado numerosas actividades, denunciando las duras condiciones de vida de muchos vecinos - tanto inmigrantes como autóctonos-y el abandono de numerosos edificios del barrio. Su actuación ha servido para articular socialmente un espacio que se transformaba con gran rapidez, haciendo confluir en proyectos comunes a colectivos y personas de procedencia muy diversa. 


\section{CUADERNO}

LOS GRANDES PROYECTOS DE DESARROLLO URBANO Y LA RECONFIGURACIÓN SOCIO-ESPACIAL DE LAS CIUDADES: EL BARRIO DE LAVAPIÉS (MADRID)

"El destino de Tabacalera es la piedra de toque que medirá el contenido efectivo de los proyectos sobre Madrid diseñados por la administración: estamos frente a ideas que pretenden crear una ciudad-marca, una ciudad-espectáculo, ciega por completo a los usos de la ciudad que hacen sus habitantes, interesada sólo en atraer dinero de turistas e inversores, en llenar los bolsillos de promotores y constructoras" (Red Lavapiés, texto de presentación de las jornadas de febrero de 2005)

El discurso de estos grupos se nutre con referencias a lo sucedido en otras ciudades europeas y norteamericanas, al significado de la economía cultural en la ciudad contemporánea o al marketing urbano. La gentrification aparece con claridad como un riesgo palpable propiciado por muchas de las actuaciones urbanas implementadas en Madrid y, en este caso particular, por la marcha del ARP de Lavapiés. La discusión sobre el futuro de la Fábrica de Tabaco simboliza el conflicto entre una ciudad pensada primordialmente para los consumos de la clase media y el turismo internacional, y las necesidades urgentes de los sectores populares, tanto autóctonos como inmigrantes. Así, la opción por los museos y el eje cultural es interpretada como la confirmación definitiva del futuro que espera a Madrid.

\section{CONCLUSIONES}

De acuerdo con las categorías manejadas páginas atrás sobre la gentrification y en general sobre los procesos de reestructuración socio-espacial, en Madrid aparecen muchas de las condiciones para que este tipo de fenómenos pueda producirse. Una economía que se ha transformado profundamente en pocos años en línea con lo experimentado por otras grandes ciudades. Cambios intensos en la estructura ocupacional que conducen, por un lado, a la expansión de nuevas clases medias urbanas y, por otro, a la llegada masiva de una población inmigrante que ha crecido rápidamente. Tras una etapa de intensa suburbanización que no parece detenerse por el momento, ciertos sectores de las clases medias y altas, en línea con lo observado en otras urbes europeas, se ven atraídos por los espacios centrales de la ciudad cargados de simbolismo histórico, y próximos a los grandes equipamientos culturales y de ocio de la metrópoli. Este no es un fenómeno completamente nuevo puesto que ya con anterioridad se habían producido algunos movimientos de menor escala, por ejemplo hacia el Madrid de los Austrias. 


\section{Fernando Díaz Orueta}

LOS GRANDES PROYECTOS DE DESARROLLO URBANO Y LA RECONFIGURACIÓN SOCIO-ESPACIAL DE LAS CIUDADES: EL BARRIO DE LAVAPIÉS (MADRID)

Asimismo, diversas áreas del centro de la ciudad presentaban a principios de los años noventa una situación de claro declive. Precisamente hacia esos espacios, junto con algunos otros de la periferia obrera, es hacia los que dirige preferentemente su atención la política de rehabilitación impulsada desde 1994 por las administraciones madrileñas. Los objetivos declarados hacen referencia a la recuperación de un patrimonio arquitectónico de gran valor y a la revitalización de estas áreas con la atracción de nueva población residente y el desarrollo del turismo. En principio, estos objetivos no deberían ser necesariamente incompatibles con los intereses de la población ya residente en la zona. Sin embargo, la orientación dada a algunas de estas intervenciones y su interpretación a la luz de la evolución seguida por el conjunto de la metrópoli, abre interrogantes importantes.

Lavapiés presentaba todos los signos del declive que podían convertirlo en acreedor de una intervención de este tipo. La concentración de infravivienda, el declive poblacional, los problemas de tráfico y consumo de drogas, etc., unido al abandono de una parte de su parque inmobiliario, configuraban una situación equiparable a la esbozada por Carpenter y Lees (1995) al caracterizar las condiciones previas al inicio de un proceso de gentrification. En ese momento, la declaración como ARP podría ser interpretada, dentro de ese esquema, como el inicio de la reinversión, implementada a gran escala desde la Administración. Aunque no se estuviera buscando voluntariamente desde el sector público la puesta en marcha de un proceso de desplazamiento y sustitución de población, lo cierto es que el tipo de políticas adoptadas podría favorecerlo. Así lo demuestran, por ejemplo, la rápida construcción de grandes equipamientos culturales mientras se posponían los más necesitados (centro de salud, escuelas, centros culturales, etc.), unas medidas de rehabilitación de vivienda insuficientes y gestionadas con lentitud que, además, no contemplaban como objetivo central a la población de menores recursos económicos del barrio (inmigrantes, ancianos, etc.) o la falta de disposición para el diálogo con el tejido asociativo, que se ha traducido en un plan en el cual la participación ciudadana ha sido ignorada.

Pero un plan de intervención no actúa sobre una foto fija. Y en Lavapiés se ha producido una transformación social muy profunda que ha venido a frenar lo que podría haber sido un rápido proceso de gentrification. En primer lugar, el asentamiento de población inmigrante en el barrio ha sido tan intenso, diverso en su composición y concentrado en el tiempo, que ha modificado muchas de las claves anteriormente manejadas. La población 


\section{CUADERNOM:M}

190

LOS GRANDES PROYECTOS DE DESARROLLO URBANO Y LA RECONFIGURACIÓN SOCIO-ESPACIAL DE LAS CIUDADES: EL BARRIO DE LAVAPIÉS (MADRID)

ha crecido por primera vez en décadas, a la vez que se rejuvenecía y se hacía más heterogénea en cuanto a su composición cultural y étnica. Y lo ha hecho en el sentido contrario al que supondría un proceso de gentrification. Por otro lado, en el barrio se ha instalado una población - menor en cantidad pero muy significativa - que podría denominarse como marginal gentrifiers (Simon 2004). Se trata de un grupo de personas solventes en lo económico (artistas, profesionales liberales, docentes, etc.) pero comprometidas con el mantenimiento del carácter socioeconómico actual de Lavapiés. En otras geografías han sido definidos como un sector de la clase media aliada a los sectores que confrontan la segregación urbana en las ciudades globales. En este caso, su aportación se hace efectiva en la colaboración con las actividades y movilizaciones de la Red y en un activismo que ha favorecido un impacto mediático positivo que, en parte, amortigua la mala imagen del barrio.

La Red en sí misma actúa también como freno a un posible proceso de gentrification. Si bien los grupos que la forman ya existían en el barrio previamente, sin embargo la declaración de ARP funcionó como un incentivo para acercar posiciones y pasar a definir con rapidez proyectos y actuaciones conjuntas. Por tanto, aunque la Administración no propició la participación, sin embargo al impulsar el Plan provocó un proceso de movilización que ha servido para cohesionar el barrio.

La foto se ha movido, dando lugar a un proceso que se ha escapado de las previsiones de los políticos y técnicos que diseñaron la intervención. Pero, en todo caso, la partida sigue jugándose. Las nuevas previsiones del Plan PERCU refuerzan todavía más la opción por la especialización turística y la atracción de nuevos residentes de mayores rentas hacia el centro urbano. La reconfiguración socio-espacial de Madrid en su conjunto sigue su curso y las operaciones contempladas para Madrid 2012 no hacen pensar en un cambio de rumbo en cuanto al modelo territorial. Así, Madrid podría seguir el camino ya recorrido por otras ciudades:

"In other European cities, competition for international sporting and cultural events, for example the Olympics, Commonwealth Games, the 'European City of Culture', city marketing and the location of cultural centers has produced a rise in gentrification of the inner city during the last ten years. The central agency of this change has been real state 


\section{Fernando Díaz Orueta}

LOS GRANDES PROYECTOS DE DESARROLLO URBANO Y LA RECONFIGURACIÓN SOCIO-ESPACIAL DE

LAS CIUDADES: EL BARRIO DE LAVAPIÉS (MADRID)

interests marketing waterside developments and associated lifestyles to urban professionals" (Gottdiener y Budd 2005: 33)

Su posición central convierte a Lavapiés en un espacio muy afectado por los procesos de reestructuración metropolitana. A través del análisis de este barrio se hacen visibles entrecruzadas y conectadas muchas de las tensiones que afectan globalmente a Madrid, se manifiesta la reconfiguración de su estructura de clases, se evidencian algunos de los cambios en los estilos de vida y de consumo.

En la actualidad, la disputa respecto de los usos futuros del antiguo edificio de la Tabacalera simboliza sobre el suelo de Lavapiés un nuevo episodio del proceso analizado en este artículo. Las autoridades locales justifican la puesta en marcha de un nuevo equipamiento cultural de alto nivel en la necesidad de hacer más atractivo el centro de Madrid, potenciando el llamado Paseo de las Artes. Pero en una ciudad fuertemente segregada y con uno de los mercados de vivienda más diferenciado espacialmente de Europa, el riesgo de una transformación social drástica en esta zona del centro resulta evidente.

\section{BIBLIOGRAFÍA}

ATENEO MADRILEÑISTA, 1989, Valoración del desarrollo del Plan General de Ordenación Urbana de Madrid. Madrid. Ateneo Madrileñista.

Bourdieu, Pierre, 1991, La distinción. Criterios y bases sociales del gusto. Madrid. Taurus.

BUTLER, TIM Y ROBSON, GARRY, 2003, London Calling: the middle classes and the making of inner London. Oxford. Berg.

CARPENTER, JULIET Y LEES, LORETA, 1995, "Gentrification in New York, London and Paris: an international comparison", International Journal of Urban and Regional Research, $\mathrm{n}^{\mathrm{O}} 19$, p. 286-303.

DÍAZ ORUETA, FERNANDO, 2001, Desequilibrios socio-territoriales en la Comunidadde Madriden la etapa de reestructuración económica (1975-1991). Madrid. Universidad Complutense de Madrid. EDIS, 2000, Lavapiés preferente. Síntesis del Estudio, Madrid.

EMPRESA MUNICIPAL DE LA VIVIENDA, 1999, Áreas de Rehabilitación Preferente. Interven- 


\section{CUADERNOM:M}

192

LOS GRANDES PROYECTOS DE DESARROLLO URBANO Y LA RECONFIGURACIÓN SOCIO-ESPACIAL DE LAS CIUDADES: EL BARRIO DE LAVAPIÉS (MADRID)

ciones en el centro histórico y barrios periféricos de Madrid, 1994-1999. Madrid. Ayuntamiento de Madrid. GLASS, RUTH, 1964, "Introduction”, en: Centre for Urban Studies (ed), London: Aspects of Change. Londres. MacGibbon and Kee, p. 13-42.

GOTTDIENER, MARK Y BUDD, LESLIE, 2005, Key Concepts in Urban Studies. Londres. Sage.

HAMNETT, CHRIS, 2003, Unequal City. London in the Global Arena. Londres. Routledge.

IOE, 1991, Inmigrantes extranjeros en la Comunidad de Madrid. Madrid.

KESTELOOT, CHRISTIAN, 2004, "Urban Socio-Spatial Configurations and the Future of European Cities", en Kazepov, Yuri (ed), Cities of Europe. Oxford. Blackwell, p. 123-148.

LABRADOR, JESÚS Y MERINO, Asunción, 2002, "Características y usos del hábitat que predomina entre los inmigrantes de la Comunidad Autónoma de Madrid", Migraciones, $\mathrm{n}^{\mathrm{o}} 11, \mathrm{p} .173-222$.

LEAL, JESÚS, 1987, "El boom inmobiliario madrileño: precios altos para rentas bajas", Alfozno 46.

LEAL, JESÚS, 1994, "Cambio social y desigualdad socioespacial en el área metropolitana de Madrid (1986-1991)", Economía y Sociedad, no 10, p. 61-79.

LEAL, JESÚS Y CORTÉS, LUIS, 1995, La vivienda en Madrid. Análisis espacial de la dinámica residencial en la región. Madrid. Consejería de Política Territorial.

LOGAN, JOHN Y MOLOTCH, HARVEY, 1987, Urban Fortunes. The Political Economy of Place. Los Angeles. University of California Press

LOURÉS, MARÍA LUISA, 2002, "Del concepto de monumento histórico al de patrimonio cultural", Revista de Ciencias Sociales. nº 94, p. 141-150.

LOURÉS, MARÍA LUISA, 2003, "Recualificación urbana y desigualdad social: los barrios en crisis en Europa", en Varios Autores, Pobreza urbana. Perspectivas globales, nacionales y locales. México. Gobierno del Estado de México, p. 89-106.

MARCOS, CARMEN, 1988, "La economía en la Comunidad de Madrid: de la crisis a la expansión", Papeles de Economía Española, n² 34, p. 141-154.

MARCUSE, PETER, 2002, "Depoliticizing Globalization: From Neo-Marxism to the Network Society of Manuel Castells", en Eade, John y Mele, Christopher (ed), Understanding the City. Contemporary and Future Perspectivas. Oxford. Blackwell, p. 131-158.

MÉNDEZ, RICARDO, 2004, "El suelo urbanizado se dispara un 25\% y el bosque pierde 240.000 hectáreas en una década", El País, 27 de diciembre de 2004, p. 28-29.

MINGIONE, ENZO, 2004, "Urban Social Change: A Socio-Historical Framework of 


\section{Fernando Díaz Orueta}

LOS GRANDES PROYECTOS DE DESARROLLO URBANO Y LA RECONFIGURACIÓN SOCIO-ESPACIAL DE LAS CIUDADES: EL BARRIO DE LAVAPIÉS (MADRID)

Analysis", en Kazepov, Yuri (ed), Cities of Europe. Oxford. Blackwell, p. 67-89.

PRETECEILLE, EDMOND, 2000, "Segregation, class and politics in large cities", en Bagnascho, Arnaldo y Le Galès, Patrick (ed), Cities in Contemporary Europe. Cambridge. Cambridge University Press, p. 74-97.

ROCH, FERNANDO, 2003, "La ciudad inmobiliaria y el precio de la vivienda", Papeles de la FIM, no 20, p. 115-129.

ROCH, FERNANDO, 2004, "El modelo inmobiliario español”, en Díaz Orueta, Fernando y Lourés, María Luisa (ed), Desigualdad social y vivienda. Alicante. ECU, p. 31-52.

SASSEN, SASKIA, 1991, The Global City: New York, London, Tokyo. Princeton. Princeton University Press.

SASSEN, SASKIA, 2003, Contrageografías de la globalización. Género y ciudadanía en los circuitos transfronterizos. Madrid. Traficantes de Sueños.

SIMON, PATRICK, 2004, "Gentrification of Old Neighborhoods and Social Integration in Europe", en Kazepov, Yuri (ed), Cities of Europe. Oxford. Blackwell, p. 210-232.

SMITH, NEIL, 1996, The new urban frontier. Gentrification and the revanchist city. Londres. Routledge. ZUKIN, SHARON, 1982, Loft Living. Culture and Capital in Urban Change. Baltimore. John Hopkins University III. 
